\section{LA ESPIRAL DE LA CREDIBILIDAD EN LOS MODELIS DE CALIDAD DE LAS ADMINISTRACIONES PÍBLICAS LOCALES}

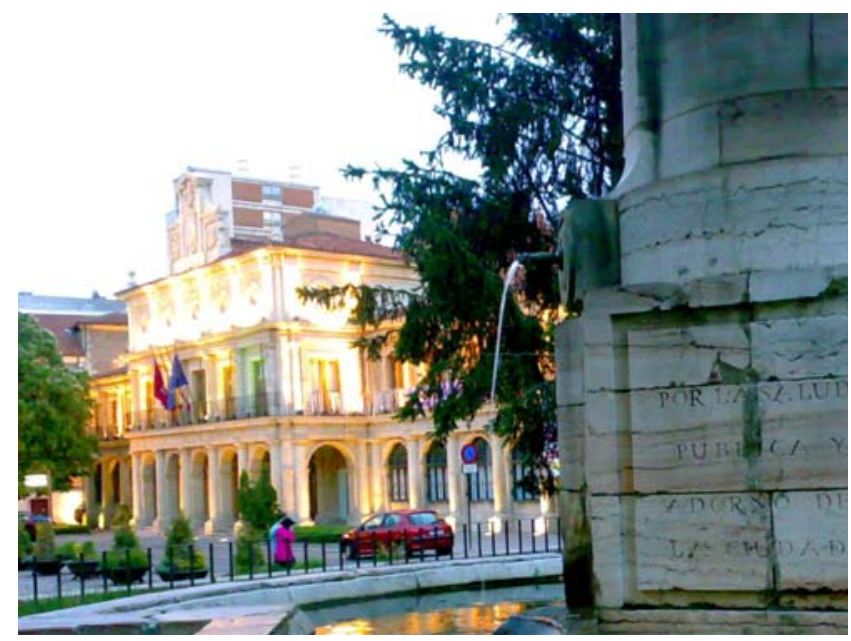

Pecvnia, Monográfico (2010), pp. 25-43

Pablo Gutiérrez Rodríguez ${ }^{1}$ pablo.gutiérrez@unileon.es

José L. Vázquez Burguete jose-luis.vazquez@unileon.es

José L. Placer Galán jose-luis.placer@unileon.es

Universidad de León

Resumen

En el actual contexto de las administraciones públicas locales, sometidas a un proceso de modernización y actualización constante en los últimos años, el concepto de calidad constituye el principal instrumento para la búsqueda del progreso de los servicios y de la competitividad. Dentro de este marco, es necesario conocer los factores determinantes de la calidad del servicio público local percibido por los ciudadanos y la repercusión de ésta sobre la satisfacción y la credibilidad. Consecuencia de la relación existente entre las variable mencionadas, la credibilidad se configura como elemento de medida de los resultados procedentes de la aplicación de las diferentes política públicas y como elemento acelerador del proceso de mejora de la calidad de estas entidades públicas.

Palabras clave: Calidad; senvicio público; satisfacción; credibilidad; gobiernos locales; ciudadano.

\title{
Abstract
}

Nowadays public administrations are involved in a constant process in terms of modernization of theirs structures and updating, the concept of quality is the mainly instrument for looking for progress in services and competitiveness. Within the public sector framework, it's

1 Facultad de Ciencias Económicas y Empresariales, Campus de Vegazana, s/n, 24071-León (España). 
necessary to know factors that determine perceived service quality in local governments by citizens and relationship between quality and satisfaction. As a result of this correspondence, credibility is going to be the element for measuring results from different public politics applied and like an accelerator for improving quality process in public organizations.

\section{Keywords Calidad; senvicio público; satisfacción; credibilidad; gobiernos locales; ciudadano.}

\section{Introduc ción}

Existe, a día de hoy, una clara preocupación por el tamaño y funcionamiento de los sectores públicos con el fin de fomentar la productividad y la eficiencia económica. Esto nos lleva a afirmar que la reforma del sector público se ha convertido en fundamental dentro de la línea de actuación de todos los gobiernos, lo que conlleva comenzar a trabajar en la gestión de la calidad, repensando la actuación de las organizaciones alrededor del concepto de satisfacción del cliente interno y externo. Precisamente, uno de los retos actuales es encontrar fórmulas para que el cliente externo de las administraciones públicas, el ciudadano, participe en la gestión administrativa discutiendo las medidas a adoptar, definiendo prioridades y fiscalizando el quehacer del gobierno y de sus diversas direcciones administrativas. De este modo, puede decirse que una de las principales aportaciones de la filosofía de la calidad es la ubicación del ciudadano en un lugar central del debate, así como la correlación de esta filosofía con una administración abierta y orientada a la eficacia externa. Todo ello no hace otra cosa que destacar la necesidad de escuchar la voz del ciudadano, con los objetivos principales de conseguir una administración más participativa, más ágil y eficaz, más transparente y próxima, centrada en la ciudadanía. Son estos últimos los que permiten tomar consciencia de la relevancia de los gobiernos locales y regionales, que se configuran como los mejores instrumentos para poder alcanzar los objetivos planteados, gracias a la cercanía con los ciudadanos y su obligación de conseguir ser más competentes y eficaces en la prestación de los servicios a los ciudadanos.

Todo este planteamiento no puede nacer sin tener en cuenta la aparición de posibles problemas. Entre otros, se señalan, la excesiva preocupación por la productividad y la eficacia, que trae como consecuencia que el papel del ciudadano se reduzca al de usuario de los servicios, tal y como lo es en su relación con las empresas del sector privado, es decir, con una capacidad de intervención real en las decisiones de la administración y en sus políticas muy limitada; o que el intento de satisfacer los deseos individuales vulnere el interés general. No es menos importante analizar los posibles cambios que la organización de los entes locales puede sufrir en los próximos años y que puede alterar la relación entre los usuarios y la administración local. La reflexión, tanto sobre la razón de ser de las organizaciones públicas -el servicio al interés general- como de las especificidades de las mismas, puede contribuir a solucionar estos problemas conceptuales. Deben proporcionar, asimismo, las claves para adaptar la filosofía y herramientas de la calidad a las necesidades organizativas de las administraciones y al logro de la eficacia, la calidad y la democracia, tan reclamadas hoy por los nuevos ciudadanos.

Así, el objetivo prioritario de este trabajo es la valoración de los factores determinantes de la calidad del servicio público local percibido por los ciudadanos y sus repercusiones sobre su satisfacción y cre- 
dibilidad, como instrumento final de medida y de reafirmación del éxito alcanzado en la gestión pública. A tal efecto, es preciso delimitar un marco espacial objeto de estudio, que está formado por los pequeños municipios de Castilla y León. La importancia de la determinación de estos factores se basa en que la calidad es el mecanismo más adecuado para contribuir a la mejora de los recursos humanos, la productividad y la adaptación de las necesidades sociales, en una época de modernización y orientación al ciudadano en que viven los entes públicos.

\section{Factores de la calidad en el servicio público}

Analizando la definición de calidad del servicio, existen dos aportaciones importantes que se centran en la percepción que el cliente tiene de la calidad recibida por parte de la empresa: el "modelo de la imagen" de Grönroos (1990: 41) y el "modelo de los gaps" de Parasuraman, Zeithaml y Berry (1985). Ambos modelos suponen que la calidad percibida es el resultado de la comparación que el cliente hace entre el servicio esperado y el servicio recibido. En principio, cuanto más altas sean las expectativas, siendo el servicio recibido el mismo, menor será la calidad percibida.

Estos modelos ayudarán a crear una escala de medida para esta variable de estudio, teniendo en cuenta las peculiaridades propias de los entes locales. Es posible distinguir, según Grönroos, entre calidad "técnica", que tiene que ver con el diseño del servicio -lo que el cliente recibe-, y calidad "funcional" -la forma en que el cliente lo recibe-. Ambos componentes de la calidad están mediatizados por la imagen de la organización. El sistema funciona de manera que, por ejemplo, si el proveedor de los servicios es bueno, se le perdonan sus fallos, pero si los errores son graves, esto repercute en la imagen, de modo que, a peor imagen, el efecto de cualquier error es mayor. Este enfoque de división de la calidad, a partir de las percepciones de los ciudadanos o usuarios de los servicios públicos, es el fundamento de nuestro trabajo. Una descripción del proceso se muestra en la Figura 1.

\section{Figura 1}

\section{Modelo de calidad percibida de servicio}

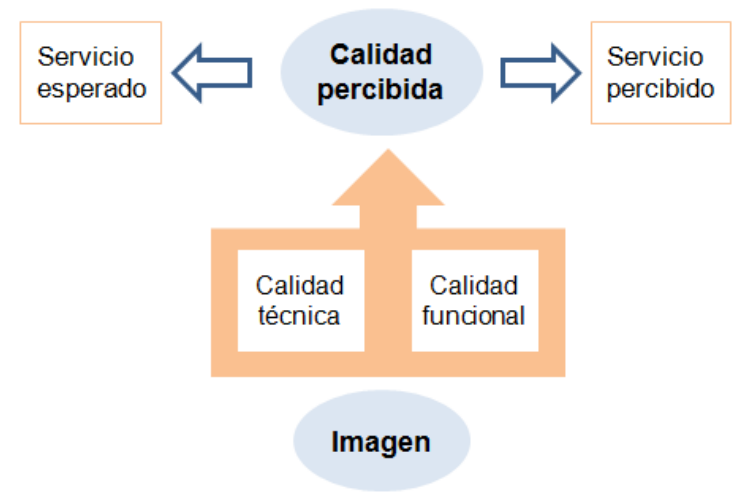

Fuente: Adaptado de DeSarbo et al. (1994: 204). 
A partir de las aportaciones de la literatura, se puede plantear cuáles son los factores más importantes que influyen sobre la calidad de la prestación en los servicios públicos. De esta manera, y de acuerdo con los planteamientos de Sancho (1999), se puede definir un decálogo de factores en lo que a calidad del servicio público se refiere. Este decálogo se agrupa en los aspectos técnicos, funcionales y de la imagen (Figura 2).

La obtención de estos factores es comparable y asimilable a la utilización de la escala SEVQUAL (empatía, capacidad, seguridad, fiabilidad y tangibles), que nos ayudará a conseguir los ítems más adecuados para cada factor. En el caso concreto de los gobiernos locales, la utilización de la escala SERVQUAL -la más utilizada - ha sido muy variada, empleándose en una amplia diversidad de contextos, ámbitos y situaciones, desde sectores de actividad industrial hasta en servicios prestados por entidades de carácter no lucrativo.

No obstante, con relación a la aplicación de esta metodología en el campo del servicio en los gobiernos locales, el número de antecedentes es muy limitado: Scott y Shieff (1993); Dalrymple et al. (1995); Donnelly et al. (1995); Wisniewski y Donnelly (1996); Gaster (1996); Donnelly y Shiu (1999); Wisniewski (2001) y Gutiérrez et al. (2009).

En las investigaciones realizadas por la práctica totalidad de los autores, la metodología empleada consiste en utilizar la escala de cinco factores con 22 ítems. Se ha analizado, como planteamiento inicial, esta metodología de escala, aunque teniendo en cuenta las peculiaridades de los gobiernos locales derivadas de su propia naturaleza pública, pero respetando las ideas y la metodología de la escala original.

\section{Figura 2}

\section{Modelo de evaluación de la calidad de los servicios públicos}

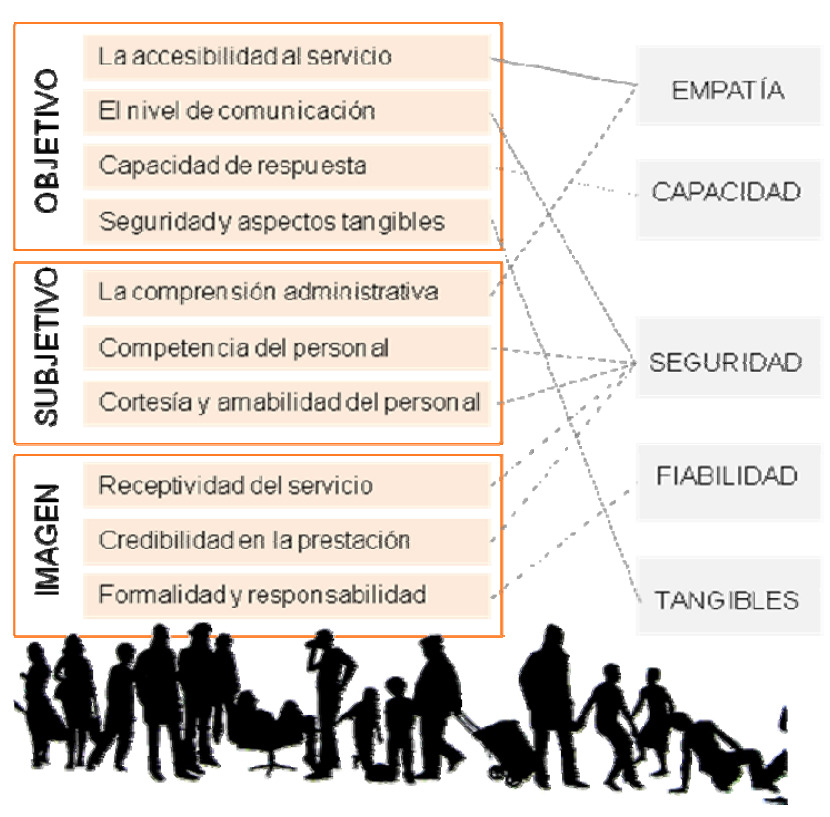

Fuente: Elaboración propia en base a Sancho (1999). 
En resumen, nuestro objetivo prioritario es la valoración de los factores determinantes de la calidad del servicio público local percibido por los ciudadanos y sus repercusiones sobre su satisfacción y credibilidad. Los beneficios de conseguir tal objetivo son comprobar la adecuación de la oferta del servicio a la demanda, detectar aquellos aspectos concretos donde la organización presenta tanto fortalezas como debilidades y facilitar la realización de estudios sobre la evolución tanto de las expectativas de los clientes como de sus percepciones (Fernández, 2000: 66). A tal efecto, es preciso delimitar un marco espacial objeto de estudio, que, como se comentó, está formado por los pequeños municipios de Castilla y León.

\section{La satisfacción y la credibilidad}

La satisfacción puede ser considerada como fundamental en la prestación de los servicios. Su importancia se debe a su incidencia sobre el bienestar del consumidor individual, sobre los beneficios de las empresas y sobre los objetivos de las empresas y entidades públicas y no lucrativas que buscan y trabajan por y para la satisfacción.
Si se analiza su etimología, la palabra satisfacción proviene del latín "satis" (suficiente) y "facere" (hacer o producir). No obstante, si lo que se lleva a cabo es una revisión de la literatura (Yi, 1990), buscando un acuerdo general para una definición de la satisfacción del consumidor, no es tan fácil, como pudiera parecer, encontrar un consenso.

La relación entre la calidad y la satisfacción se basa en la discrepancia entre las expectativas y las percepciones, que da lugar a la satisfacción (Vázquez, Rodríguez y Díaz, 1996) -Figura 3- o a la insatisfacción, tal y como se conoce del paradigma disconfirmatorio, de la misma manera que se calcula la calidad de servicio (Gil, 1996). Dicho de otro modo, la satisfacción se obtiene cuando el consumidor compara las percepciones con las expectativas; si el resultado que se obtiene de esta comparación supera las expectativas del consumidor (disconformación positiva), se obtiene como resultado un consumidor satisfecho; en caso contrario, donde el resultado percibido es menor que las expectativas (disconformación negativa), se tiene que el consumidor estará insatisfecho (Oliver, 1980).

\section{Figura 3}

\section{Relación entre satisfacción y calidad de servicio percibida}

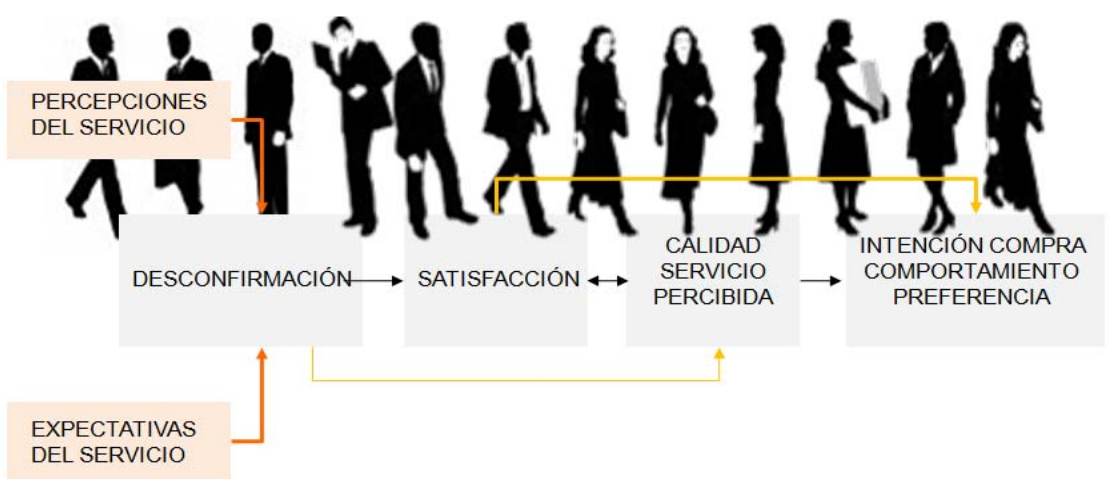

Fuente: Vázquez, Rodríguez y Díaz (1996). 
Fornell proclama que la satisfacción del cliente influye en el comportamiento de compra, así el consumidor satisfecho tiende a ser un consumidor leal, pero el consumidor leal no es necesariamente un consumidor satisfecho. Andreassen (1994) propone que la satisfacción y la lealtad están influenciadas por la credibilidad.

Lo que sucede es que los usuarios pueden ser leales debido a la existencia de barreras o a la ausencia de alternativas reales, además según Kandampully y Suhartanto (2000) la lealtad de los consumidores se basa en la intención de este de volver a comprar el mismo servicio siempre que sea posible. Todo esto lleva a replantear el concepto de lealtad al aplicarlo al ámbito público, de este modo, la lealtad, en el ámbito de las administraciones públicas, toma otra dimensión o se analiza según otros aspectos, que aparecen a continuación, los cuales adapten la lealtad a los gobiernos locales.

Surge el concepto de confianza o credibilidad que ha recibido una especial atención en la literatura de marketing, debido a la notable influencia que ejerce en la consecución de relaciones duraderas y rentables (Morgan y Hunt, 1994). Tradicionalmente, ha sido considerada como un conjunto de creencias dispuestas en la mente del individuo y derivadas de las percepciones que este tiene acerca de determinados atributos que caracterizan a la marca, oferta, personal de ventas o al propio establecimiento donde se comercializan los productos (Ganesan, 1994). Este conjunto de creencias ha sido dividido en distintas dimensiones, por lo que la confianza suele ser considerada como un constructo multidimensional, en el que se diferencian la honestidad y benevolencia percibidas en los comportamientos de la otra parte (De Wulf y OdekerkenSchoröder, 2003). La honestidad es la creencia en que la otra parte mantiene su palabra, cumple sus promesas y es sincera, esto es, "la creencia de un miembro del canal de que el socio es fiable, mantiene su palabra, cumple sus promesas y obligaciones, y es sincero" (Geyskens, Steenkamp y Kumar, 1998). Por otro lado, la confianza es la creencia en la benevolencia de los actos del socio. En este sentido, la confianza es la fe en que una parte se interesa por los intereses y el bienestar de la otra, y que aquélla está motivada en la búsqueda de ganancias conjuntas (Anderson, Lodish y Weitz, 1987; Crosby, Evans y Cowles, 1990) y sin la intención de llevar a cabo comportamientos oportunistas (Larzelere y Huston, 1980). Además, la benevolencia hace referencia a que no se tomarán acciones imprevistas que pudieran tener impactos negativos en el socio (Anderson y Narus, 1990; Andaleeb, 1995).

En todo caso, algunos investigadores han contemplado variantes en la naturaleza de las dimensiones que caracterizan a la confianza. De hecho, es relativamente habitual encontrar referencias a la competencia percibida en la otra parte (Sako y Helper, 1997; Roy, Dewit y Aubert, 2001). Esta última dimensión hace referencia a la destreza que se percibe en la otra parte (Mayer, Davis y Shoorman, 1995; Sirdeshmukh, Singh y Sabor, 2002). Concretamente, la competencia es el grado en el que los consumidores perciben que el proveedor dispone de las habilidades y el conocimiento necesario (Coulter y Coulter, 2002). Otra definición, que reafirma el posterior trabajo empírico, es la de Sheldom (1996), para quien la credibilidad se logra conforme a cuatro factores básicos, la atención y la empatía, la percepción de la competencia y habilidades de la fuente, la percepción de la honestidad y la percepción del compromiso y la dedicación. En resumen, estos factores redundan en la relación que existirá entre la calidad percibida y la credibilidad, gracias a la consecución de satisfacción del cliente. Pero pocos autores han profundizado de manera empírica en las relaciones entre cali- 
dad, satisfacción y credibilidad. Uno de los trabajos más destacados, aunque en el marco teórico, pertenece a Van de Walle, Van Roosbroek y Bouckaert (2008) quienes tratan de establecer los determinantes de la credibilidad.
El resultado de todo este planteamiento teórico, y tras depurar y establecer la representatividad y validez de los ítems, es el conjunto de preguntas del cuestionario planteado a los ciudadanos de los municipios (Tabla 1).

\section{Tabla 1}

\section{Cuestionario utilizado}

\section{Calidad técnica del servicio}

\section{Calidad funcio- nal del servicio}

\section{Elementos gene- rales o de la imagen del servicio}

\section{Aspectos de la} satisfacción, la calidad y la credibilidad
La disposición de las oficinas del ayuntamiento permite hablar con el personal del ayuntamiento fácilmente (comodidad).

Los formularios, papeleo o comunicados del ayuntamiento son fáciles de entender (el contenido).

El teléfono (fax, Internet) es una buena comunicación con el ayuntamiento (otras vías de comunicar).

Cuando se comunica algo, este comunicado hace referencia a aspectos de interés para el ciudadano.

Las comunicaciones (o comunicados) utilizan un lenguaje sencillo con el ciudadano.

En el ayuntamiento, los empleados siempre están dispuestos a ayudar a los ciudadanos, comprometidos.

La zona de atención del público del ayuntamiento está en buenas condiciones.

Cuando prometen hacer algo lo cumplen en el plazo solicitado.

Es comprensible el funcionamiento (quién es quién y cómo funciona) del ayuntamiento.

Sabe a quién dirigirse para solucionar sus problemas.

El personal posee las habilidades y conocimientos para desempeñar el puesto de trabajo asignado.

Los empleados informan de los pasos a seguir para solicitar cualquier servicio (o queja).

Los empleados del ayuntamiento son amables y respetuosos.

Los empleados tienen en cuanta la situación personal (no ofrecen a todos lo mismo, hay circunstancias)

Las acciones o servicios (o nuevos servicios) de su ayuntamiento han cambiado por las ideas de los ciudadanos

Las quejas son tenidas en cuenta.

Los empleados le inspiran confianza.

Los empleados tienen los recursos necesarios para desarrollar los servicios.

Basándome en lo que conozco, clasifico la calidad global de este ayuntamiento como excelente.

Cuando comparo los servicios de este ayuntamiento con los de otro similar, la calidad de este ayuntamiento es mayor.

En términos generales, estoy satisfecho con la calidad de este ayuntamiento.

Los servicios del municipio los debe prestar el ayuntamiento y no otra administración (Junta o Estado).

El ayuntamiento ofrece credibilidad o confianza a los ciudadanos.

Fuente: Elaboración propia. 
Del amplio conjunto de indicadores relacionados con las variables latentes, se han introducido en el modelo los que figuran la Tabla citada anteriormente. Para su selección se han tenido en cuenta los criterios que dicta la teoría económica teniendo en cuenta los límites que incorpora el encontrarnos en una administración pública. Las variables latentes, que formarán la variable "calidad percibida" son la "calidad funcional" o los "aspectos funcionales" y la "calidad técnica" o "aspectos técnicos", los de la "imagen", influyen y conforman ambos aspectos de la calidad.

\section{Metodología y datos}

El modelo se ha estimado empleando como fuente las encuestas realizadas a los residentes, mayores de 16 años, de los municipios de Castilla y León con un tamaño no superior a cinco mil habitantes. La unidad de análisis requiere que el encuestado haya recibido o participado en la prestación de algún servicio de su ayuntamiento en los últimos tres meses, siendo la fecha de realización de la encuesta en Febrero y Marzo del año 2005. Se realizaron un total de 400 encuestas validas, lo que supone un error muestral $\pm 5,00$ (con un intervalo de confianza del 95,5\%).

\section{Tabla 2}

\section{Ficha técnica}

\begin{tabular}{|c|c|}
\hline Ámbito geográfico & Regional (Castilla y León) \\
\hline Objeto evaluado & $\begin{array}{l}\text { Evaluación del servicio que prestan las entidades locales } \\
\text { de menos de } 5.000 \text { habitantes. }\end{array}$ \\
\hline Población & $\begin{array}{l}\text { Ciudadanos residentes en dichos municipios mayores de } \\
16 \text { años. }\end{array}$ \\
\hline Tamaño de la población & 856.684 habitantes mayores de 16 años \\
\hline Método de obtención de la información & $\begin{array}{l}\text { Entrevistas piloto, cuestionarios y Anuarios de la Junta } \\
\text { de Castilla y León }\end{array}$ \\
\hline Número de cuestionarios válidos & 400 \\
\hline Intervalo de confianza & $95,5 \%$ \\
\hline Error muestral & $\pm 5,00 \%$ \\
\hline Trabajo de campo & $\overline{\text { Desde el }} 12$ de febrero hasta el 10 de marzo \\
\hline Tratamiento de la información & $\begin{array}{l}\text { Programa para modelización de ecuaciones estructurales } \\
\text { (PLS): SmartPLS y PLS-VB }\end{array}$ \\
\hline
\end{tabular}

El procedimiento de estimación empleado es el de los Mínimos Cuadrados Parciales (PLS) introducido por Wold (1982). Este método iterativo permite la estimación de los parámetros de un modelo multiecuacional como el presentado, donde se pueden encontrar una serie de variables no observables o latentes que son estimadas como combinación lineal de una serie de indicadores o variables manifiestas. El empleo de mínimos cuadrados parciales (PLS) frente a otro método de máximo verosimilitud para la estimación de las relaciones estructurales, queda justificado por las propiedades de este método expuestas por Chin (1998). El uso del método PLS se basa en las mínimas exigencias respecto a las escalas de medida, tamaño muestral y establecimiento de hipótesis de distribución de las perturbaciones aleato- 
rias. La principal distinción entre PLS y otros enfoques de estimación basados en la covarianza, como LISREL, es debida a la finalidad del modelo de ecuaciones estructurales. El primero de los enfoques es más adecuado para la estimación de modelos susceptibles de aplicación y predicción, además evita los problemas de indeterminación y soluciones no admisibles. Mientras que, el segundo, es más apropiado cuando se cuenta con una base teórica consistente y se quiere verificar una teoría, pero hay una pérdida de precisión debido a problemas de indeterminación, es el apropiado para los modelos donde la principal preocupación es la estimación de las relaciones estructurales. Mientras, el PLS estima las variables latentes como combinación de los indicadores, evitando la posible indeterminación y obteniendo un valor concreto para los resultados finales. También, debido al empleo de un algoritmo iterativo consistente en estimaciones mínimo cuadráticas, la identificación no es un obstáculo para los modelos recursivos ni se exige hipótesis sobre la función de distribución de las variables.

Además, el tamaño de la muestra puede ser pequeño, dándose como regla genérica que el tamaño puede ser igual a diez veces el número de indicadores formativos o a diez veces el mayor número de coeficientes estructurales dirigidos a una construcción particular en la relación estructural. En este estudio se cumple esta regla sobradamente. Además PLS, según Wold (1985), no tiene competidor para muestras grandes y modelos complejos de variables latentes, donde la atención pasa de variables e indicadores individuales a un conjunto de ellos. Sin embargo, este método de estimación presenta algún inconveniente, el primero es que los estimadores no son óptimos, respecto a la consistencia y posibilidad de ser insesgados, debido a que es un método de información limitada, (no se supone normalidad). Pero asintóticamente los estimado- res son correctos cuando trabajamos con muestras grandes y cuando el número de indicadores por variable latente es elevado. Otro inconveniente es que los errores estándar han de ser estimados por remuestreo, mediante Bootstrapping o Jackknifing.

Una vez que hemos seleccionado los ítems que conforman la encuesta, es interesante establecer la validez de constructo. Para ello estaría el análisis de la validez factorial, el cual, se considerada por algunos autores como una herramienta muy útil (García, Gil y Rodríguez, 2000: 17), al afirmar que "el análisis factorial se nos revela también como una herramienta que puede utilizarse para la validación de la escala".

La Tabla 3 muestra la matriz de componentes obtenida mediante el método de Componentes Principales, que permite extraer los factores que más varianza explican, es decir, que acumulan más información de un conjunto grande de variables. De ahí que el factor que mejor explique una dimensión analizada (el que represente mayor variabilidad) se convierta en el primer componente principal, el que mejor explique la segunda dimensión, será el segundo componente, y así sucesivamente.

Los valores que aparecen en el interior de la tabla pueden interpretarse como índices de correlación entre cada factor y cada variable (llamados pesos o cargas). Se observa que la mayoría de los ítems tienen pesos iguales o por encima de 0,5 mientras que el resto de los factores son superiores a 0,4 .

De la interpretación de esta matriz, hay que decir que esta escala es básicamente unidimensional, ya que, como se ha comentado, todos los ítems tienen saturaciones bastante elevadas en el primer factor. La unidimensionalidad, supuesto subyacente y requisito esencial para cons- 
truir una escala aditiva (Hair et al. 1999: 105), implica que los distintos ítems de la escala se relacionan unos con otros representando un único concepto.
En resumen, los resultados obtenidos tras aplicar los diversos test a los que se ha sometido el instrumento de medida confirman la existencia de validez de constructo.

\section{Tabla 3}

\section{Matriz de componentes}

\begin{tabular}{|c|c|c|c|c|}
\hline \multirow{2}{*}{\multicolumn{2}{|c|}{ Ítems }} & \multicolumn{3}{|c|}{ Componentes } \\
\hline & & 1 & 2 & 3 \\
\hline 1 & Aspecto técnico 1 & 0,765 & $-0,119$ & $-0,031$ \\
\hline 2 & Aspecto técnico 2 & 0,613 & $-0,082$ & 0,290 \\
\hline 3 & Aspecto técnico 3 & 0,420 & 0,296 & 0,400 \\
\hline 4 & Aspecto técnico 4 & 0,455 & 0,361 & 0,376 \\
\hline 5 & Aspecto técnico 5 & 0,559 & $-0,304$ & 0,324 \\
\hline 6 & Aspecto técnico 6 & 0,411 & $-0,373$ & $-0,019$ \\
\hline 7 & Aspecto técnico 7 & 0,506 & $-0,463$ & 0,364 \\
\hline 8 & Aspecto funcional 1 & 0,619 & 0,280 & 0,061 \\
\hline 9 & Aspecto funcional 2 & 0,620 & 0,140 & 0,223 \\
\hline 10 & Aspecto funcional 3 & 0,552 & 0,174 & 0,328 \\
\hline 11 & Aspecto funcional 4 & 0,788 & $-0,133$ & $-0,132$ \\
\hline 12 & Aspecto funcional 5 & 0,759 & $-0,057$ & $-0,198$ \\
\hline 13 & Aspecto funcional 6 & 0,721 & $-0,136$ & $-0,345$ \\
\hline 14 & Aspecto funcional 7 & 0,486 & $-0,034$ & $-0,430$ \\
\hline 15 & Aspecto imagen 1 & 0,416 & 0,370 & $-0,157$ \\
\hline 16 & Aspecto imagen 2 & 0,588 & 0,275 & $-0,246$ \\
\hline 17 & Aspecto imagen 3 & 0,775 & 0,000 & $-0,330$ \\
\hline 18 & Aspecto imagen 4 & 0,428 & 0,262 & 0,026 \\
\hline 19 & Calidad global 1 & 0,764 & $-0,118$ & $-0,030$ \\
\hline 20 & Calidad global 2 & 0,613 & $-0,081$ & 0,290 \\
\hline 21 & Satisfacción 1 & 0,420 & 0,295 & 0,399 \\
\hline 22 & Credibilidad 1 & 0,454 & 0,361 & 0,375 \\
\hline 23 & Credibilidad 2 & 0,559 & $-0,304$ & 0,324 \\
\hline
\end{tabular}

Fuente: Elaboración propia.

\section{Resultados del modelo y sus relaciones}

Todo este planteamiento teórico podría verse plasmado en el planteamiento de las siguientes hipótesis que se van a tratar de confirmar:
$\mathrm{H}_{1}$ : La calidad percibida está determinada por los aspectos funcionales, técnicos y de la imagen.

$\mathrm{H}_{2}$ : La credibilidad está determinada por la calidad percibida tanto de manera directa como indirecta (a través de la satisfacción).

$\mathrm{H}_{3}$ : La mejora en la credibilidad revierte en la mejora de la propia calidad percibida. 


\section{Figura 4}

\section{Resumen de las hipótesis planteadas}

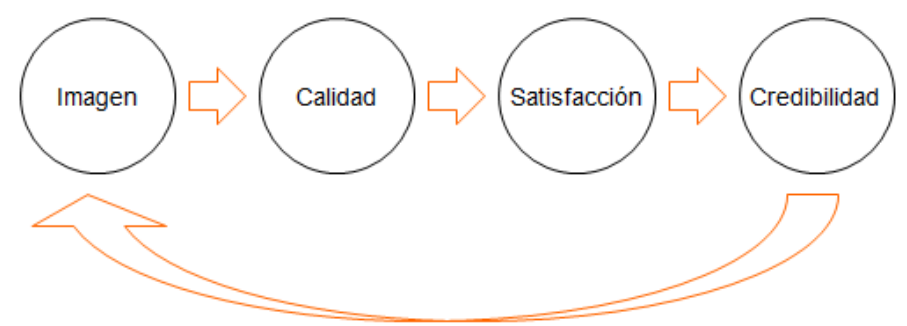

Fuente: Elaboración propia.

Uno de los métodos que se podría utilizar para establecer la fiabilidad de los contenidos es la Varianza Media Extraída (AVE). Este método es más conservador que el coeficiente rho, por lo que es más severo a la hora de garantizar la validez de los contenidos (Fornell y Larker, 1981). Aunque se habla de contenido, lo que realmente se mide a través de AVE es la validez del discriminante, o lo que es lo mismo, en que medida cada uno de los constructos son diferentes de los otros. En la práctica esto se traduce en conocer su capacidad para medir distintos conceptos (Rivard et al., 1994). Para ello, se utilizará el AVE. Este método trata de medir como la varianza del constructo debe participar más que otros constructos en el modelo.
De este modo, en el caso de las variables estandarizadas:

$$
\begin{aligned}
& \sum\left(\Lambda_{\mathrm{i}}^{2}\right) \\
& \mathrm{AVE}=\mathrm{N}^{\mathrm{o}} \text { de indicadores de la } \\
& \text { variable }
\end{aligned}
$$

Para certificar que la varianza entre un constructo y sus medidas es mayor que la varianza entre el constructo y los otros constructos, en el análisis PLS se observa la matriz de correlaciones de las variables latentes, donde la diagonal es reemplazada por la raíz cuadrada de los valores de AVE. Los mayores valores de la diagonal, comparados con el resto de constructos confirma la buena validez del discriminante, en concreto, si se analiza el modelo planteado (Tabla 4).

\section{Tabla 4}

\section{Matriz de Correlaciones y Raíz Cuadrada de AVE}

\begin{tabular}{|lccc}
\hline \multicolumn{1}{c}{ Constructo } & Aspectos técnicos & Aspectos funcionales & Aspectos generales \\
\hline Aspectos técnicos & 0,730 & 0,713 & 0,633 \\
\hline Aspectos funcionales & 0,713 & 0,744 & 0,680 \\
\hline Aspectos de la imagen & 0,632 & 0,680 & 0,704 \\
\hline Fuente: Elaboración propia. & & & \\
\hline
\end{tabular}

Por lo tanto, se podría afirmar que existe una validez de discriminante para las variables que constituyen los constructos "calidad técnica", "calidad funcional" y "aspectos de la imagen". 


\section{Figura 5}

\section{Salida del modelo planteado}

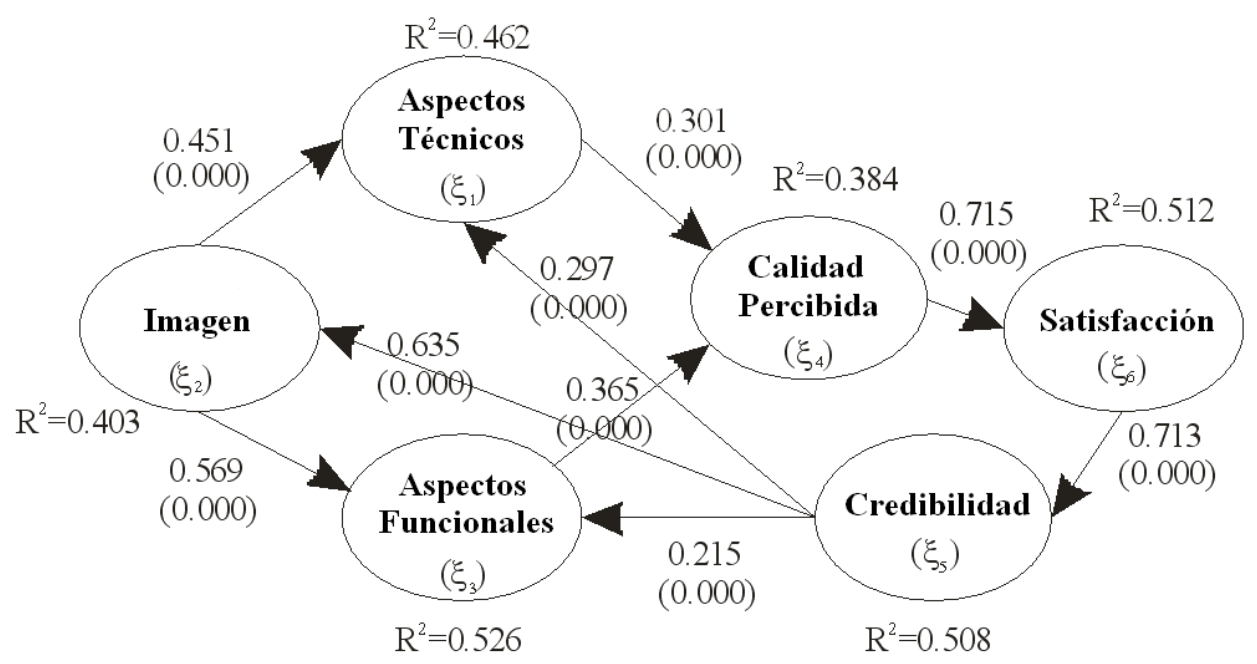

Fuente: Elaboración propia.

Una vez desarrollado el modelo, la siguiente fase consiste en analizar su estructura mediante los dos siguientes procedimientos:

- Análisis del poder explicatorio de los constructos independientes.

PLS analiza esta situación a través del análisis de los valores del R2 asociados con varios constructos dependientes; por ello presenta una explicación sobre la proporción de la varianza explicada. Igual que en la regresión, el valor de R2 representa el porcentaje de variación de las variables dependientes que puede ser explicado por las variables independientes del modelo. No hay un umbral de valor que sugiera que el modelo no tiene sentido, pero la comparación de los valores R2 con los posibles modelos alternativos puede ser de extremada ayuda. De cualquier modo, un valor por debajo de 0,2 sugeriría la necesidad de replantear el modelo. Los valores, para el modelo planteado ronda el valor 0,5 (Figura 5).

- Análisis del tamaño y la significación de las relaciones entre los coeficientes.

Para el análisis de la importancia de las relaciones entre las diferentes variables del modelo son importantes no sólo las relaciones directas sino también los efectos indirectos sobre las variables y determinar, de este modo, los efectos globales (véase Tabla 5).

Como anteriormente hemos explicado, los aspectos funcionales, técnicos y de la imagen son variables latentes constituidas por un conjunto de cuestiones que se ven reflejadas en dichas variable. Su relación -de estas variables latentes- con la variable calidad es bastante importante, dado que los tres aspectos forman en constructo de calidad, ya sea de manera directa o indirecta. 


\section{Tabla 5}

\section{Efectos directos e indirectos}

\begin{tabular}{lcc}
\multicolumn{1}{c}{ Relaciones } & Efectos Directos & Efectos Indirectos \\
Aspectos técnicos $\rightarrow$ Calidad & 0.301 & - \\
\hline Aspectos funcionales $\rightarrow$ Calidad & 0.365 & - \\
Aspectos de la imagen $\rightarrow$ Aspectos técnicos & 0.451 & - \\
\hline Aspectos de la imagen $\rightarrow$ Aspectos funcionales & 0.569 & - \\
\hline Aspectos de la imagen $\rightarrow$ Calidad & - & $0.301 * 0.451+0.365^{*} 0.569$ \\
\hline Calidad $\rightarrow$ Satisfacción & 0.715 & - \\
\hline Satisfacción $\rightarrow$ Credibilidad & 0.713 & - \\
Credibilidad $\rightarrow$ Aspectos técnicos & 0.297 & - \\
Credibilidad $\rightarrow$ Aspectos funcionales & 0.215 & - \\
Credibilidad $\rightarrow$ Aspectos de la imagen & 0.635 & \\
Fuente: Elaboración propia. & &
\end{tabular}

Uno de los elementos de análisis fundamental es la relación entre la calidad y la satisfacción. Además de existir una alta significación, podemos decir que existe una alta relación. El efecto directo entre estas variables es de 0,715 , o lo que es lo mismo, si conseguimos elevar la percepción de la calidad en una unidad, el aumento de la satisfacción del ciudadano será de 0,715 unidades (en una escala Likert entre 1 y 5). Lo mismo sucede en la influencia de la satisfacción sobre la credibilidad $(0,713)$. No son tan grandes las influencias de la credibilidad sobre los factores funcionales y técnicos $(0,215 \mathrm{y}$ 0,297 respectivamente) pero la influencia se puede medir como suma de todos los efectos (también incluimos la relación entre credibilidad e imagen de 0,635). Con estos resultados, podemos afirmar que las relaciones planteadas tienen una fuerte correspondencia. Lo que nos permite establecer la importancia de la calidad sobre la satisfacción y la credibilidad. Además, con estos resultados, se observan que, los establecidos como determinantes de la calidad, lo son de manera cierta.

\section{Ajuste global del modelo}

Wold (1982) propone el uso de un método para medir la relevancia predictiva: el test de Stone-Geisser (Stone, 1974; Geisser, 1974). Este test, básicamente, realiza una estimación jackknife (eliminación de manera constante de una variable) de las varianzas residuales, mientras que los jackknife de los errores estándar de los puntos estimados pueden ser obtenidos por elementos. La idea general es omitir un caso cada período para luego reestimar los parámetros del modelo sobre los casos restantes $y$, entonces, reconstruir o predecir los valores omitidos usando los parámetros reestimados. Este ejercicio puede ser realizado a través del estadístico $Q^{2}$ propuesto por Ball, aplicado para $i=1,2$, ...., $\mathrm{n}$ casos y una regresión múltiple con $\mathrm{k}$ regresiones, $\mathrm{Q}^{2}$ se plantea como:

$$
\mathrm{Q}^{2}=1-\frac{\sum_{n}\left(\mathrm{Yi}-\sum \mathrm{X}_{k i} \mathrm{~b}_{k(i)}\right)^{2}}{\sum_{n}\left(\mathrm{Yi}-\mathrm{Y}_{\cdot(i)}\right)^{2}}=1-\frac{E}{O}
$$


Donde bk(i) es el conjunto de coeficientes de la regresión obtenidos cuando el iésimo caso es omitido mientras que Y.(i) es la media de la variable dependiente obtenida sin los casos i-ésimos. Se puede ver entonces que $\mathrm{Q}^{2}$ no es más que el análogo jackknife de la familia de los $R^{2}$. Sin embargo, a pesar de esta característica cabe destacar dos aspectos de este estadístico: primero, que puede incrementarse si alguna predicción se elimina de la ecuación (esto es, se elimina el ruido que realizan algunas predicciones inestables) $y$, segundo, que el valor puede tornarse negativo indicando, en este caso la confusión o mala dirección del modelo planteado. Este estadístico no asume o presupone ningún tipo de distribución y puede utilizarse para la evaluación del poder predictivo del modelo planteado.

En este caso, y para las variables dependientes, los valores son positivos y se recomienda que sean mayores a 0,2 .

\section{Tabla 6}

\section{Valores de R2 y $\mathbf{Q}^{2}$ y $\mathbf{G}_{\mathrm{j}}$}

\begin{tabular}{lcc} 
Construct & $\mathbf{R}^{2}$ & $\mathbf{Q}^{2}$ \\
\hline$\eta_{1}=\mathrm{f}\left(\xi_{1}, \xi_{3}\right)$ & 0.384 & 0.170 \\
\hline$\eta_{2}=\mathrm{f}\left(\xi_{4}\right)$ & 0.512 & 0.430 \\
$\eta_{3}=\mathrm{f}\left(\xi_{6}\right)$ & 0.508 & 0.230 \\
$\eta_{4}=\mathrm{f}\left(\xi_{2}, \xi_{5}\right)$ & 0.526 & 0.186 \\
$\eta_{5}=\mathrm{f}\left(\xi_{5}\right)$ & 0.403 & 0.122 \\
$\eta_{6}=\mathrm{f}\left(\xi_{2}, \xi_{5}\right)$ & 0.462 & 0.124
\end{tabular}

Fuente: Elaboración propia.

El programa estadístico utilizado (PLS-VB) tiene la particularidad de establecer una herramienta para la elección del modelo más adecuado. Se elegirá el modelo que mejor describa las relaciones entre las variables exógenas y endógenas. Para la identificación del modelo más estable, se necesita especificar primero las " $r$ " variables objetivo, las cuales asumidas, entre las $(p-q)$ endógenas, van a ser de primordial interés en relación al problema que se plantea.
Estas variables -las cuales corresponden, para mayor sencillez, a las últimas " $r$ " ecuaciones del modelo- serán consideradas como la referencia básica en el criterio de adopción adoptado para evaluar la representación del modelo. El modelo se expresa a través de los $b_{j k}$ coeficientes, siendo los valores latentes los valores observados. En concreto, el criterio adoptado sugiere que se elija el que alcance el menor valor de la siguiente función:

$$
\mathrm{G}\left(\mathrm{g}_{p-r+1}, \ldots, \mathrm{g}_{p}\right)=\sum_{j=p-r+1}^{\mathrm{p}}\left(\ln \hat{\sigma}_{j}^{2}+\mathrm{g}_{j} \frac{\mathrm{Ln} n}{\mathrm{n}}\right)=\sum_{j=p-r+1}^{p} \mathrm{G}_{j}
$$


Donde:

$\widehat{\sigma}^{2}(j=p-r+1, p)$ son las varianzas de los residuos de la regresión referente a las "r" variables objetivo Yj de la correspondiente regresión, siendo $g_{j} \geq 0$; sabiendo que si $g_{j}=0$ se tiene que $\sigma_{2 j}=\operatorname{Var}(Y j)$. Los valores obtenidos tienen especial relevancia cuando son comparados con los resultados de otros modelos planteados o reespecificados.

\section{Conc lusiones}

La relación directa y notoria entre la calidad percibida y la satisfacción es un hecho que la literatura ha demostrado, tanto en el sector privado como en el público. No lo es tanto, aunque a nivel teórico si se plantea, la relación con la credibilidad de los ciudadanos. El uso de escalas y técnicas de medición de la calidad en las administraciones públicas abre la posibilidad al desarrollo de políticas públicas más precisas en la búsqueda de la satisfacción del cliente local, el ciudadano, y la utilización de la credibilidad como medida del éxito conseguido. La participación ciudadana se convierte en la manera de conseguir una modernización de los servicios públicos, ya que aporta una reflexión fundamentada en el sólido análisis teórico y analítico acerca de la relación entre la Administración y el ciudadano en el contexto de la prestación de servicios públicos municipales.

De esta manera, este estudio nos permite, con cierto grado de representatividad y significación, determinar los factores que influyen en la calidad y, por razón de las fuertes relaciones existentes, en la satisfacción de los ciudadanos. Cabe destacar, además, los efectos circulares conseguidos por medio de la credibilidad que nos animan a intentar mejorar la calidad percibida ya que, además de los efectos directos sobre la satisfacción, conseguiremos efectos posteriores y positivos provenientes de la credibilidad, creando una espiral ya que los efectos se van mitigando tras la realización de las acciones de mejora de la calidad.
En cualquier caso, una de las principales conclusiones, es la determinación de los elementos o factores de la percepción de la calidad, tal y como aparece en el modelo planteado, son los aspectos técnicos, los aspectos funcionales y los aspectos genéricos de los gobiernos locales. En resumen podemos concluir que:

- Se observa la menor puntuación que los aspectos generales obtienen en comparación con los otros dos componentes de la calidad. Ello nos hace reflexionar sobre la posibilidad de tratar de desarrollar políticas para la mejora de estas variables, debido al mayor margen existente de mejora.

- En relación a los aspectos técnicos, para conseguir una mejora en la satisfacción del ciudadano de los entes locales, los ayuntamientos deben asegurarse de que sus puntos de atención al ciudadano estén actualizados y representen una imagen que atraiga a sus mercados objetivos (Baker, Wagner y Singer, 2003). Así, no puede olvidarse que la implantación de las nuevas tecnologías en la actualidad es un servicio básico para cualquier ciudadano, independientemente del tamaño del municipio en que resida.

- El tercer grupo de elementos que se han descubierto como determinantes de la calidad percibida, y por lo tanto de la satisfacción, son los aspectos funcionales. Este factor se basa en los recursos humanos de los entes locales. Los trabajadores, por medio de sus conocimientos sobre los procedimientos, su amabilidad y el análisis de la situación personal de la persona que tienen delante, pueden hacer variar de forma muy significativa la satisfacción del "cliente" público. Por otro lado, es fundamental que las entidades locales trans- 
mitan una imagen de eficacia en tanto que contribuye a la prestación de un servicio público de calidad. Esta eficacia está asociada en gran medida al cumplimiento de plazos que todo procedimiento burocrático lleva aparejado.

- Finalmente, realizar una llamada de atención acerca de la importancia -y al mismo tiempo escasez- del desarrollo de estudios sobre la actuación de los ayuntamientos en la provisión de bienes y servicios públicos a la ciudadanía. Su relevancia responde, en primer lugar, a los intensos cambios que está sufriendo la sociedad española actual $y$, en segundo lugar, al intenso grado de descentralización del que ha sido objeto el sector público español, que no se ha reflejado en el incremento de las competencias de gasto e ingreso en las corporaciones locales. Cuanta mayor sea la calidad del servicio público percibida por el ciudadano, menor será su oposición a su financiación

e, incluso, esto redundará en una mayor cooperación entre la Administración y la ciudadanía para lograr la adecuación entre la expectativa del ciudadano y el servicio prestado por el sector público. Si a esto sumamos la fuerte repercusión sobre la satisfacción, tanto los ciudadanos como los políticos se encuentran con una herramienta que puede serles útil a ambos.

Es necesario también recalcar, entre las limitaciones existentes, que los resultados obtenidos se derivan del análisis de los ciudadanos con la administración local en un determinado marco espacial formado por los municipios de Castilla y León con una población inferior a cinco mil habitantes. Esta situación viene a poner de relieve cómo estos resultados no son extrapolables, si bien en algunos términos pueden ser aplicados a otros ayuntamientos, en toda su extensión. Lógicamente, las corporaciones locales de esta u otra comunidad autónoma con mayor número de habitantes se enfrentan básicamente al mismo reto: la satisfacción del ciudadano, aunque con otras casuísticas (transporte público, servicio de aguas, recogida de basura,...).

\section{Bibliografía}

Andaleeb, S.S. (1995). "Dependence relations and the moderating role of trust: implications for behavioral intentions in marketing channels". International Journal of Research in Marketing, vol. 12, n. 2, 157-172.

Anderson, E.; Lodish, L. y Weitz, B. (1987). “Resource allocation behavior by channel members". Journal of Marketing Research, vol. 24, n. 1, pp. 85-97.

Anderson, J.C. y Narus, A. (1992). "A model of distributor firm and manufacture firm working partnerships". Journal of Marketing, vol. 54, n. 1, pp.42-58.

Andreassen, T.W. (1994). "Satisfaction, loyalty and reputation as indicators of customer orientation in the public sector". International Journal of Public Sector Management, vol. 7, n. 2, pp. 16-34.

Baker, L.; Wagner, T.H. y Singer, S. (2003). "Use of the Internet and E-mail for Health Care Information Results from a National Survey". Journal of the American Medical Association, vol. 289, n. 18, pp. 2400-2406.

Chin, W.W. (1998). "The partial least squares approach for structural equation modeling”. In, Modern methods for business research. G.A. Marcoulides (Ed.), Mahwah, NJ: Lawrence Erlbaum Associates, pp. 295-336. 
Coulter, K. y Coulter, R. (2002). "Determinants of trust in a service provider: the moderating role of length of relationship". Journal of Services Marketing, vol. 16, n. 1, pp. 35-50.

Crosby, L.A.; Evans, K.R. y Cowles, D. (1990). "Relationship quality in services selling: An interpersonal influence perspective”. Journal of Marketing, vol. 54, n. 3, pp. 6881.

Dalrymple, J.F.; Donnelly, M.; Wisniewski, M. y Currie, A.C. (1995). “Measuring service quality in local government". In, Kanji G.K. (Ed.), Proceedings of the First World Congress on TQM. Londres: Chapman and Hall, pp. 263-266.

De Wulf, K. y Odekerken-Schröder, G. (2003). “Assessing the impact of a retailer's relationship efforts on consumer' attitudes and behaviour". Journal of Retailing and Consumer Services, vol. 10, n. 2, pp. 95-108.

DeSarbo, W.S.; Huff, L.; Rolandelli, M.M. y Choi, J. (1994). “On the measurement of perceived service quality. A conjoint analysis approach". In, Service quality. New directions in theory and practice. Rust, R.T. y Oliver, R.L. (Eds.). Thousand Oaks, California: SAGE Publications Inc., pp. 201-222.

Donnelly, M. y Shiu, E. (1999). "Assessing service quality and its link with value for money in a UK local authority's housing repairs service using the SERVQUAL approach". Total Quality Management, vol. 10, n. 4/5, pp. 498-506.

Donnelly, M.; Wisniewski, M.; Dalrymple, J.F. y Curry, A.C. (1995). “Measuring service quality in local government: The SERVQUAL approach". International Journal of Public Sector Management, vol. 8, n. 7, pp. 15-20.

Fernández Barcala, M. (2000). "Validación de SERVQUAL como instrumento de medida de la calidad del servicio bancario". Revista Europea de Dirección y Economía de la Empresa, vol. 9, n. 1, pp. 57-70.

Fornell, C. (1982). "A national customer satisfaction barometer: The Swedish experience". Journal of Marketing, vol. 56, n. 1, pp. 6-21.

Ganesan, S. (1994). "Determinants od long-term orientation in buyer-seller relationship". Journal of Marketing, vol. 58, n. 2, pp. 1-19.

García, E.; Gil, J. y Rodríguez, G. (2000). Análisis factorial. Colección Cuadernos de Estadística. Madrid: La Muralla.

Gaster, L. (1996). “Quality services in local government: a bottom-up approach”. Journal of Management Development, vol. 15 n. 2, pp. 80-96.

Geisser, S. (1974). "A predictive approach to the random effects model". Biometrika, vol. 61, n. 1, pp. 101-107.

Geyskens, I.; Steenkamp, J. y Kumar N. (1998). "Generalizations about trust in marketing channel relationships using meta-analysis". International Journal of Research in Marketing, vol. 15, n. 3, pp. 223-248.

Gil, I. (1996). "La evaluación de la calidad de servicio percibida y las limitaciones de la herramienta Servqual". Revista Europea de Dirección y Economía de la Empresa, vol. 5, n. 2, pp. 19-32.

Grönroos, C. (1990). Service management and marketing: Managing the moments of truth in service competition. Lexington, MA: Lexington Books. 
Gutiérrez, P.; Vázquez, J.L.; Vaughan, R. y Edwards, J. (2009). "Quality dimensions in the public sector: Municipal services and citizen's perception". International Review on Public and Non Profit Marketing, vol. 6, n. 1, pp 75-90.

Hair, J.F.; Anderson, R.E.; Tatham, R.L. y Black, W.C. (1999). Análisis multivariante. $5^{a}$ ed. Madrid: Prentice Hall.

Kandampully, J. y Suhartanto, D. (2000). “Customer loyalty in the hotel industry: The role of customer satisfaction and image". International Journal of Contemporary Hospitality Management, vol. 12, n. 6, pp. 346-351.

Larzelere, R. y Huston, T. (1980). "The dyadic trust scale: Toward understanding interpersonal CONF in close relationships". Journal of Marriage and the Family, vol. 42, n. 3, pp. 595-604.

Mayer, R.; Davis, J. y Shoorman, F. (1995). "An integrative model of organizational trust". Academy of Management Review, vol. 20, n. 3, pp. 709-734.

Morgan, R. y Hunt, S. (1994). "The commitment - trust theory of relationship marketing". Journal of Marketing, vol. 58, n. 3, pp. 20-38.

Oliver, R.L. (1980). "A cognitive model of the antecedents and consequences of satisfaction decisions". Journal of Marketing Research, vol. 17, n. 4, pp. 460-469.

Parasuraman, A.; Zeithaml, V.A. y Berry, L.L. (1985). “A conceptual model of service quality and its implications for future research". Journal of Marketing, vol. 49, n. 4, pp. 41-50.

Rivard, S.; Poirier, G.; Raymond, L. y Bergeron, F. (1994). "Development of a measure to assess the quality of user-developed applications". In, Proceedings of the TwentySeventh Annual Hawaii International Conference on System Sciences, J.F. Nunamaker y R.H. Sprague (Eds.). Hawaii, vol. IV, pp. 522-531.

Roy, M.; Dewit, O. y Aubert, B. (2001). "The impact of interface usability on trust in web retailers". Internet Research: Electronic Networking Applications and Policy, vol. 11, n. 5, pp. 388-398.

Sako, M. y Helper, S. (1997). "Determinants of trust in supplier relations: Evidence from the automotive industry in Japan and the United States". Journal of Economic Behaviour and Organization, vol. 34, n. 3, pp. 387-417.

Sancho Royo, D. (1999). Gestión de servicios públicos: Estrategias de marketing y calidad. Madrid: Ed. Tecnos.

Scott, D. y Shieff, D. (1993). "Service quality components and group criteria in local government". International Journal of Service Industry Management, vol. 4, n. 4, pp. 42-53.

Sheldom, K. (1996). Credibility is risky business: An interview with Vincent T. Covello. Ph.D. Commun. World 13.

Sirdeshmukh, D.; Singh, J. y Sabor, B. (2002). "Consumer trust, value and loyalty in relational exchanges”. Journal of Marketing, vol. 66, n. 1, pp. 15-37.

Stone, M. (1974). "Cross validatory choice and assessment of statistical predictions". Journal of the Royal Statistical Society, Series B 36, pp. 111-133. 
Van de Walle, S.; Van Roosbroek, S. y Bouckaert, G. (2008). "Trust in the public sector: is there any evidence for a long-term decline?". International Review of Administrative Sciences, vol. 74, n. 1, pp. 47-64.

Vázquez Casielles, R.; Rodríguez del Bosque, I. y Díaz Martín, A.M. (1996). “Estructura multidimensional de la calidad de servicio en cadenas de supermercados: desarrollo y validación de la escala CALSUPER". Documento de trabajo 119/96. Facultad de Ciencias Económicas y Empresariales. Universidad de Oviedo.

Wisniewski, M. y Donnelly, M. (1996). "Measuring service quality in the public sector: The potential for SERVQUAL”. Total Quality Management, vol. 7, n. 4, pp. 357-366.

Wisniewski, M. (2001). "Using SERVQUAL to assess customer satisfaction with public sector services". Managing Service Quality, vol. 11, n. 6, pp. 995-1001.

Wold, H. (1982). "Soft modeling. The basic design and some extensions". In, Systems under indirect observation. Jöreskog, K.G. y Wold, H. (Eds.). Amsterdam, North Holland.

Yi, Y. (1990). "A critical review of consumer satisfaction”. In, Review of Marketing. Chicago: American Marketing Association, pp. 68-123. 